\title{
Recent patents in metagenomics
}

Patent number Description

US 9,372,959 Systems and methods for assembly of metagenomic sequences. In one embodiment, a plurality of metagenomic sequences is represented in threedimensional space to obtain a plurality of sequence vectors. Based on plurality of the sequence vectors, a cuboid having a plurality of grids is defined in the three-dimensional space such that it encompasses the plurality of metagenomic sequences. Further, the plurality of metagenomic sequences is assembled into one or more contigs based on traversal of the plurality of grids. In one implementation, the one or more contigs are assembled such that a contig includes metagenomic sequences probably originating from the same genome.

US 9,080,211 Methods, compositions and kits for using a transposase and a transposon end for generating extensive fragmentation and 5 '-tagging of double-stranded target DNA in vitro, then using a DNA polymerase for generating $5^{\prime}$ - and 3 '-tagged single-stranded DNA fragments without performing a PCR amplification reaction, wherein the first tag on the 5 '-ends exhibits the sequence of the transferred transposon end and optionally, an additional arbitrary sequence, and the second tag on the 3 '-ends exhibits a different sequence from the sequence exhibited by the first tag. The method is useful for generating 5 '- and 3 '-tagged DNA fragments for use in a variety of processes, including processes for metagenomic analysis of DNA in environmental samples, copy number variation (CNV) analysis of DNA, and comparative genomic sequencing (CGS), including massively parallel DNA sequencing.

US 8,906,656 Polypeptides identified among translated coding sequences from a metagenomic cow rumen database, and shown to provide xylose isomerase activity in yeast cells. The xylose isomerase activity can complete a xylose utilization pathway so that yeast can use xylose in fermentation, such as xylose in biomass hydrolysate.

US 8,586,336 A novel xylose isomerase nucleotide sequence obtained from a bovine rumen fluid metagenomic library that also provides the amino acid sequence encoded by the nucleotide sequence, and a vector and a transformant containing the nucleotide sequence. When the xylose isomerase is expressed, a host cell is endowed with the capability of converting xylose into xylulose, and the xylulose is further metabolized by the host cell. Therefore, the host cell can take the xylose as a carbon source for growth. The xylose isomerase from a new source is expressed with high activity in Saccharomyces cerevisiae and is a mesophilic enzyme with optimal temperature of $60^{\circ} \mathrm{C}$.

US 8,478,544 Systems and methods capable of characterizing populations of organisms within a sample. The characterization may utilize probabilistic matching of short strings of sequencing information to identify genomes from a reference genomic database to which the short strings belong. The characterization may include identification of the microbial community of the sample to the species and/or sub-species and/or strain level with their relative concentrations or abundance. May enable rapid identification of organisms including both pathogens and commensals in clinical samples, and the identification may be achieved by a comparison of many (e.g., hundreds to millions) metagenomic fragments, which have been captured from a sample and sequenced, to many (e.g., millions or billions) of archived sequence information of genomes (i.e., reference genomic databases).

US 7,910,522 Methods for the identification or cloning of polynucleotides encoding a selected phenotype, particularly from environmental DNA. In a specific embodiment, the method comprises (i) cloning environmental DNA fragments into $E$. coli cloning vectors to produce a metagenomic library, (ii) identifying or selecting cloning vectors in the library which contain DNA fragments having a particular characteristic of interest, (iii) modifying the identified or selected cloning vectors into shuttle or expression vectors for transfer and integration in a selected host cell, (iv) transferring the modified cloning vectors into the selected host cell and (v) identifying or cloning the DNA fragments contained in the modified cloning vectors which encode the selected phenotype in the selected host cell.

US 7,867,747 A novel protease, a polynucleotide encoding the protease, and a fibrinolytic agent comprising the same. The protease is obtained from a new gene source by using metagenomic library technology, and can replace the conventional fibrinolytic agent.

US 7,853,408 A method of designing oligonucleotides that permits designing specific oligonucleotides for the identification of a determined sequence in a metagenomic sample.

CosmosID (College

Park, MD, USA)
Grunenwald HL,

Epicentre

Technologies

(Madison, WI, USA)

Caruccio N, Jendrisak J,

Dahl G
$7 / 14 / 2015$
Inventor

Mande SS,

Ghosh TS, Mehra V
Date

6/21/2016

E I du Pont

de Nemours

and Company

(Wilmington, DE,

USA)

(Jinan, China)
Chen Z, Kelly KJ, Ye RW

$12 / 9 / 2014$

Bao X, Shen Yu, Ge R

$11 / 19 / 2013$
Colwell RR, Livingston BT, 7/2/2013

Jakupciak D,

Hasan NA, Jakupciak JP,

Brenner DM $\begin{array}{ll}\text { Libragen (Toulouse, } & \text { Nalin R, Pujic P, } \\ \text { France) } & \text { Tuphile K, Gillet B }\end{array}$

Korea Ocean

Research and

Kyunggido, S. Korea) Jang M-K

Biosigma (Colina,

Chile)
Maass Sepulveda AE,

Aravena Duarte AO,

Gonzalez Canales MA,

Martinez Aguilera S,

Parada Valdecantos PA

Ehrenfeld Stolzenbach KN
$1 / 11 / 2011$

$12 / 14 / 2010$

Source: United States Patent and Trademark Office (http://www.uspto.gov). 